\title{
NUMERICAL MODELLING OF THE TRANSIENT HEAT TRANSPORT IN A THIN GOLD FILM USING THE FUZZY LATTICE BOLTZMANN METHOD WITH $\alpha$-CUTS
}

\author{
Alicja Piasecka Belkhayat, Anna Korczak \\ Institute of Computational Mechanics and Engineering \\ Silesian University of Technology, Poland \\ alicja.piasecka@polsl.pl,anna.korczak@polsl.pl
}

\begin{abstract}
In this paper a description of heat transfer in one-dimensional crystalline solids is presented. The fuzzy lattice Boltzmann method based on the Boltzmann transport equation is used to simulate the nanoscale heat transport in thin metal films. The fuzzy coupled lattice Boltzmann equations for electrons and phonons are applied to analyze the heating process of thin metal films via a laser pulse. Such an approach in which the parameters appearing in the problem analyzed are treated as constant values is widely used. Here, the model with fuzzy values of relaxation times and an electron-phonon coupling factor is taken into account. The problem formulated has been solved by means of the fuzzy lattice Boltzmann method using the $\alpha$-cuts and the rules of directed interval arithmetic. The application of $\alpha$-cuts allows one to avoid complicated arithmetical operations in the fuzzy numbers set. In the final part of the paper the results of numerical computations are shown.
\end{abstract}

Keywords: fuzzy lattice Boltzmann method, $\alpha$-cuts, directed interval arithmetic

\section{Introduction}

The estimation of heat transfer processes proceeding in metal micro-domains (e.g. thin metal films) subjected to an ultrafast laser pulse is of vital importance in microtechnology applications. It should be pointed out that taking into account the extreme temperature gradients, extremely short duration and the domain dimensions expressed in nanometers, the macroscopic heat conduction equation basing on the Fourier law cannot be used [1-3]. The generalization of this law resulting from the introduction of the delay time between the heat flux and temperature gradient (relaxation time $\tau_{q}$ ) leads to the well known Cattaneo-Vernotte equation [4] belonging to the group of hyperbolic PDE. The alternative, more general, mathematical model called the dual phase lag equation (DPLE) takes into account two delay times concerning both the heat flux and the temperature gradient (the thermalization time $\tau_{T}$ ). The DPLE contains a second order time derivative and higher order mixed derivative in both time and space (e.g. [5-8]). The other approach to the micro-scale 
heat transfer analysis involves the use of two-temperature models. The two-temperature hyperbolic (parabolic) model consists of equations describing the temporal and spatial evolution of the lattice and electrons temperatures, the lattice and electron heat fluxes [9-11].

In this paper the thermal processes proceeding in the micro-domains are analyzed using the fuzzy lattice Bolzmann method $[12,13]$. The heat transfer problems are usually solved assuming that the equations appearing in the mathematical model and all parameters in these equations are deterministic. Such an assumption does not give an exact image of the thermal processes met in the engineering practice. It seems more natural to take into account uncertainties related, for example, to the material parameters. Here, the fuzzy lattice Bolzmann method is presented using the $\alpha$-cuts of fuzzy numbers with the approach of the directed interval arithmetic [14]. The interval values of relaxation times and boundary conditions are taken into account. The application of $\alpha$-cuts allows one to avoid very complicated arithmetical operations in the fuzzy numbers set because the $\alpha$-cuts are closed intervals. In this case, the mathematical operations are defined according to the rules of the directed interval arithmetic performed for every $\alpha$-cut, which is treated as an interval number. In this arithmetic, a set of proper intervals is extended by improper intervals, and all arithmetic operations and functions are also extended. The main advantage of the directed interval arithmetic upon the usual interval arithmetic is that the obtained temperature intervals are much narrower and their width does not increase in time.

\section{Boltzmann transport equation}

During the heating of thin metal films via laser pulse the electrons are energized and they subsequently transfer the energy to phonons via coupling between them. The Boltzmann transport equations for the coupled model (1D problem) with two kinds of carriers: electrons $(e)$ and phonons $(p h)$ take the following form [15]:

$$
\begin{gathered}
\frac{\partial f_{e}}{\partial t}+\mathbf{v}_{e} \cdot \nabla f_{e}=\frac{f_{e}^{0}-f_{e}}{\tau_{e}}+g_{e} \\
\frac{\partial f_{p h}}{\partial t}+\mathbf{v}_{p h} \cdot \nabla f_{p h}=\frac{f_{p h}{ }^{0}-f_{p h}}{\tau_{p h}}+g_{p h}
\end{gathered}
$$

where $f_{e}, f_{p h}$ are the carrier distribution functions, $f_{e}^{0}, f_{p h}{ }^{0}$ are the equilibrium distribution functions given by the Bose-Einstein statistic for phonons [16] and Fermi-Dirac statistic for electrons [17], $\mathbf{v}_{e}, \mathbf{v}_{p h}$ are the frequency-dependent carrier propagation speeds, $\tau_{e}, \tau_{p h}$ are the frequency-dependent carrier relaxation times and $g_{e}, g_{p h}$ are the carrier generation rates due to external sources such as laser heating. 
The BTEs (1) and (2) can be transformed into equivalent carrier energy density equations [15]

$$
\begin{gathered}
\frac{\partial e_{e}}{\partial t}+\mathbf{v}_{e} \cdot \nabla e_{e}=\frac{e_{e}^{0}-e_{e}}{\tau_{e}}+Q_{e} \\
\frac{\partial e_{p h}}{\partial t}+\mathbf{v}_{p h} \cdot \nabla e_{p h}=\frac{e_{p h}{ }^{0}-e_{p h}}{\tau_{p h}}+Q_{p h}
\end{gathered}
$$

where $e_{e}, e_{p h}$ are the carrier energy densities, $e_{e}^{0}, e_{p h}{ }^{0}$ are the equilibrium carrier energy densities and $Q_{e}, Q_{p h}$ are the carrier energy sources related to a unit of volume. The equations (3) and (4) must be supplemented by the adequate boundary-initial conditions.

The electron and phonon energy densities at their equivalent nonequilibrium temperatures are given by the formulas

$$
\begin{gathered}
e_{e}\left(T_{e}\right)=\left(n_{e} \frac{\pi^{2}}{2} \frac{k_{b}^{2}}{\varepsilon_{F}}\right) T_{e}^{2} \\
e_{p h}\left(T_{p h}\right)=\left(\frac{9 \eta k_{b}}{\Theta_{D}^{3}} \int_{0}^{\Theta_{D} / T_{p h}} \frac{z^{3}}{\exp (z)-1} \mathrm{~d} z\right) T_{p h}{ }^{4}
\end{gathered}
$$

where $T_{e}, T_{p h}$ are the carrier temperatures, $k_{b}$ is the Boltzmann constant, $\varepsilon_{F}$ is the Fermi energy, $n_{e}$ is the electron density, $\Theta_{D}$ is the Debye temperature of the solid, $\eta$ is the number density of oscillators [15].

The electron and phonon energy sources are calculated using the following expressions [15]:

$$
\begin{gathered}
Q_{e}=Q^{\prime}-G\left(T_{e}-T_{p h}\right) \\
Q_{p h}=G\left(T_{e}-T_{p h}\right)
\end{gathered}
$$

where $Q^{\prime}$ is the power density deposited by the external source function associated with the laser irradiation [18] and $G$ is the electron-phonon coupling factor which characterizes the energy exchange between electrons and phonons.

\section{Fuzzy numbers}

The ground of the mathematical rules used in this paper is given by the fuzzy set theory. This approach is not common in solving heat transfer problems and that is why some of the definitions used in this concept must be explained [19]. 
First of all, the definition of a fuzzy set will be introduced. The fuzzy set $\tilde{A}$ in a non empty universal set $\mathbb{X}(\tilde{\mathrm{A}} \subseteq \mathbb{X})$ can be expressed by a set of pairs consisting of the elements $x \in \mathbb{X}$ and a certain degree of pre-assumed membership $\mu_{\tilde{\mathrm{A}}}(x)$ of the form

$$
\tilde{\mathrm{A}}=\left\{\left(x, \mu_{\tilde{\mathrm{A}}}(x)\right) ; x \in \mathbb{X}\right\}
$$

where function $\mu_{\tilde{\mathrm{A}}}(x)$ is defined as

$$
\mu_{\tilde{\mathrm{A}}}: \mathbb{X} \rightarrow[0,1]
$$

In fuzzy sets, each element is mapped to $[0,1]$ by membership function $\mu_{\tilde{\AA}}(x)$, where $[0,1]$ means real numbers between 0 and 1 (including 0,1 ). Consequently, a fuzzy set is a 'vague boundary set' compared with a crisp set.

For every $x \in \mathbb{X}$ can be considered three types of membership to the fuzzy set $\tilde{\mathrm{A}}$ :

- $\mu_{\tilde{\mathrm{A}}}(x)=1$ - full membership to the fuzzy set, $x \in \tilde{\mathrm{A}}$,

- $\mu_{\tilde{\mathrm{A}}}(x)=0$ - lack of membership to the fuzzy set, $x \notin \tilde{\mathrm{A}}$,

- $0<\mu_{\tilde{\mathrm{A}}}(x)<1$ - partial membership to the fuzzy set.

The $\alpha$-cut set $\tilde{\mathrm{A}}$ in universal set $\mathbb{X}$ is made up of members whose membership is not less than $\alpha$ for every $\alpha \in[0,1][20]$

$$
\tilde{\mathrm{A}}_{\alpha}=\left\{x \in \mathbb{X}: \mu_{\tilde{\mathrm{A}}}(x) \geq \alpha\right\}
$$

The value $\alpha$ is arbitrary and this $\alpha$-cut set is a crisp set. This set is determined by the following characteristic function:

$$
\chi_{\tilde{\mathrm{A}}_{\alpha}}= \begin{cases}1 & \text { for } \mu_{\tilde{\mathrm{A}}}(x) \geq \alpha \\ 0 & \text { for } \mu_{\tilde{\mathrm{A}}}(x)<\alpha\end{cases}
$$

Every fuzzy set $\tilde{\mathrm{A}}$ can be defined as a sum of all its $\alpha$-cuts

$$
\tilde{\mathrm{A}}=\sum_{\alpha \in[0,1]} \alpha \cdot \tilde{\mathrm{A}}_{\alpha}
$$

where $\alpha \cdot \tilde{\mathrm{A}}_{\alpha}$ is a fuzzy set in the universe $\mathbb{X}$, whose membership function is the following:

$$
\mu_{\alpha \cdot \tilde{\mathrm{A}}_{\alpha}}= \begin{cases}\alpha & \text { for } x \in \tilde{\mathrm{A}}_{\alpha} \\ 0 & \text { for } x \notin \tilde{\mathrm{A}}_{\alpha}\end{cases}
$$


Arithmetical operations are generally very complicated. Among the infinite quantity of possible fuzzy sets that can be qualified as fuzzy numbers, some types of membership functions are of particular importance.

Due to its rather simple membership function of a linear type, triangular fuzzy numbers are one of the most frequently used fuzzy numbers. In this paper the triangular fuzzy numbers will be used to solve the Boltzmann transport equations specified for various mathematical models.

A triangular fuzzy number $\tilde{a}$ is a set with the following membership function [21]:

$$
\mu_{\tilde{a}}(x)=\left\{\begin{array}{lr}
0, & x<a^{-} \\
\frac{x-a^{-}}{a_{0}-a^{-}}, & a^{-} \leq x \leq a_{0} \\
\frac{a^{+}-x}{a^{+}-a_{0}}, & a_{0} \leq x \leq a^{+} \\
0, & x>a^{+}
\end{array}\right.
$$

where $a_{0}$ is the core of the number, $a^{-}, a^{+}$are the left and the right end of the number, respectively. A triangular fuzzy number can be written as $\tilde{a}=\left(a^{-}, a_{0}, a^{+}\right)$.

One of the ways to avoid very complicated arithmetic operations performed on fuzzy numbers is to apply $\alpha$-cuts of fuzzy numbers. In this case, the mathematical operations are defined according to the rules of the directed interval arithmetic performed for every $\alpha$-cut.

The $\alpha$-cut of a fuzzy number $\tilde{a}$ defined by a pair of functions $a^{-}:[0,1] \rightarrow \mathbb{R}$ and $a^{+}:[0,1] \rightarrow \mathbb{R}$ is called a set of closed intervals [22]

$$
\forall \alpha \in[0,1] \quad \tilde{a}_{\alpha}=\left[a_{\alpha}^{-}, a_{\alpha}^{+}\right]
$$

which satisfies the following conditions

$$
\begin{aligned}
& \text { 1. } a^{-}: \alpha \rightarrow a_{\alpha}^{-} \in \mathbb{R} \\
& \text { 2. } a^{+}: \alpha \rightarrow a_{\alpha}^{+} \in \mathbb{R} \\
& \text { 3. } a_{\alpha}^{-} \leq a_{\alpha}^{+}
\end{aligned}
$$

where $a^{-}\left(a^{+}\right)$is a limited, monotonic function for every $\alpha \in[0,1]$.

Every fuzzy number can be presented as a sum of all its own $\alpha$-cuts

$$
\tilde{a}=\sum_{\alpha \in[0,1]} \tilde{a}_{\alpha}
$$


The $\alpha$-cut of a triangular fuzzy number $\tilde{a}=\left(a^{-}, a_{0}, a^{+}\right)$is the set of all closed intervals in the form

$$
\forall \alpha \in[0,1] \quad \tilde{a}_{\alpha}=\left[\left(a_{0}-a^{-}\right) \alpha+a^{-},\left(a_{0}-a^{+}\right) \alpha+a^{+}\right]
$$

The decomposition of a fuzzy number allows one to make the mathematical operations on closed intervals which are $\alpha$-cuts. In this situation, complicated arithmetic operations can be omitted and it is possible to apply the interval arithmetic for every $\alpha$-cut. The mathematical operations are simplified, because they are done only on the ends of the intervals.

For the $\alpha$-cuts of two fuzzy numbers $\tilde{a}$ and $\tilde{b}$, the following mathematical operations can be defined ( $\forall \alpha \in[0,1])[22]$ :

- addition

$$
(\tilde{a}+\tilde{b})_{\alpha}=\left[a_{\alpha}^{-}+b_{\alpha}^{-}, a_{\alpha}^{+}+b_{\alpha}^{+}\right]
$$

- subtraction

$$
(\tilde{a}-\tilde{b})_{\alpha}=\left[a_{\alpha}^{-}-b_{\alpha}^{+}, a_{\alpha}^{+}-b_{\alpha}^{-}\right]
$$

- multiplication by a scalar $(k \in \mathbb{R})$

$$
k \cdot \tilde{a}_{\alpha}=\left[\min \left\{k a_{\alpha}^{-}, k a_{\alpha}^{+}\right\}, \max \left\{k a_{\alpha}^{-}, k a_{\alpha}^{+}\right\}\right]
$$

- multiplication

$$
(\tilde{a} \cdot \tilde{b})_{\alpha}=\left[\min \left\{a_{\alpha}^{-} b_{\alpha}^{-}, a_{\alpha}^{-} b_{\alpha}^{+}, a_{\alpha}^{+} b_{\alpha}^{-}, a_{\alpha}^{+} b_{\alpha}^{+}\right\}, \max \left\{a_{\alpha}^{-} b_{\alpha}^{-}, a_{\alpha}^{-} b_{\alpha}^{+}, a_{\alpha}^{+} b_{\alpha}^{-}, a_{\alpha}^{+} b_{\alpha}^{+}\right\}\right]
$$

- inverse $\left(0 \notin\left[a_{\alpha}^{-}, a_{\alpha}^{+}\right]\right)$

$$
\left(\frac{1}{\tilde{a}}\right)_{\alpha}=\left[\frac{1}{a_{\alpha}^{+}}, \frac{1}{a_{\alpha}^{-}}\right]_{\alpha}
$$

- division $\left(0 \notin\left[b_{\alpha}^{-}, b_{\alpha}^{+}\right]\right)$

$$
\left(\frac{\tilde{a}}{\tilde{b}}\right)_{\alpha}=\left[\min \left\{\frac{a_{\alpha}^{-}}{b_{\alpha}^{-}}, \frac{a_{\alpha}^{-}}{b_{\alpha}^{+}}, \frac{a_{\alpha}^{+}}{b_{\alpha}^{-}}, \frac{a_{\alpha}^{+}}{b_{\alpha}^{+}}\right\}, \max \left\{\frac{a_{\alpha}^{-}}{b_{\alpha}^{-}}, \frac{a_{\alpha}^{-}}{b_{\alpha}^{+}}, \frac{a_{\alpha}^{+}}{b_{\alpha}^{-}}, \frac{a_{\alpha}^{+}}{b_{\alpha}^{+}}\right\}\right]_{\alpha}
$$

Applying $\alpha$-cuts of the fuzzy numbers allows one to use directed interval arithmetic [13, 23]. 


\section{Fuzzy lattice Boltzmann method}

The lattice Boltzmann method is a discrete representation of the Boltzmann transport equations. The fuzzy form of the BTEs for $1 \mathrm{D}$ problems can be expressed as [24]

$$
\begin{gathered}
\frac{\partial \tilde{e}_{e}}{\partial t}+\mathbf{v}_{e} \cdot \nabla \tilde{e}_{e}=\frac{\tilde{e}_{e}^{0}-\tilde{e}_{e}}{\tilde{\tau}_{e}}+\tilde{Q}_{e} \\
\frac{\partial \tilde{e}_{p h}}{\partial t}+\mathbf{v}_{p h} \cdot \nabla \tilde{e}_{p h}=\frac{\tilde{e}_{p h}^{0}-\tilde{e}_{p h}}{\tilde{\tau}_{p h}}+\tilde{Q}_{p h}
\end{gathered}
$$

where $\tilde{e}_{e}, \tilde{e}_{p h}$ are the fuzzy values of carrier energy densities for electrons and phonons, respectively, $\tilde{e}_{e}^{0}, \tilde{e}_{p h}{ }^{0}$ are the fuzzy equilibrium carrier energy densities and $\tilde{\tau}_{e}, \tilde{\tau}_{p h}$ are the fuzzy relaxation times.

The fuzzy values of the electron and phonon energy sources are calculated using the following formulas according to the rules of directed interval arithmetic [25]

$$
\begin{gathered}
\tilde{Q}_{e}=Q^{\prime}-G\left(\tilde{T}_{e}-\tilde{T}_{p h}\right) \\
\tilde{Q}_{p h}=G\left(\tilde{T}_{e}-\tilde{T}_{p h}\right)
\end{gathered}
$$

where $G$ is the electron-phonon coupling factor.

For the one-dimensional model the discrete set of propagation velocities in two lattice directions (1 and 2) for electrons and phonons is defined as (see Fig. 1)

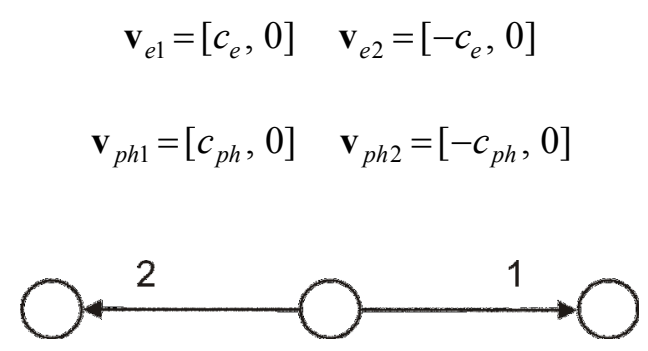

Fig. 1. One-dimensional 2-speed lattice Boltzmann model

The equations (26) and (27) should be supplemented by the boundary conditions [12], for example of the $1^{\text {st }}$ type on the left boundary and the $2^{\text {nd }}$ type on the right boundary as follows: 


$$
\begin{cases}x=0: & \tilde{e}_{e}(0, t)=e_{e}\left(\tilde{T}_{b 1}^{e}\right) \\ x=L: & \tilde{q}_{b}^{e}(L, t)=v_{e}\left[e_{e}\left(\tilde{T}_{e}\right)_{n-1}-e_{e}\left(\tilde{T}_{e}\right)_{n}\right] \\ x=0: & \tilde{e}_{p h}(0, t)=e_{p h}\left(\tilde{T}_{b 1}^{p h}\right) \\ x=L: & \tilde{q}_{b}^{p h}(L, t)=v_{p h}\left[e_{p h}\left(\tilde{T}_{p h}\right)_{n-1}-e_{p h}\left(\tilde{T}_{p h}\right)_{n}\right]\end{cases}
$$

and the initial conditions

$$
\begin{array}{ll}
t=0: & e_{e}(x, 0)=e_{e}\left(T_{0}^{e}\right) \\
t=0: & e_{p h}(x, 0)=e_{p h}\left(T_{0}^{p h}\right)
\end{array}
$$

where $\tilde{T}_{b 2}^{e}$ and $\tilde{T}_{b 2}^{p h}$ are the fuzzy values of boundary temperatures of electrons and phonons respectively, $\left(\tilde{T}_{e}\right)_{0}$ and $\left(\tilde{T}_{e}\right)_{1}$ are the fuzzy temperatures of electrons in the first and second node, $\left(\tilde{T}_{p h}\right)_{0}$ and $\left(\tilde{T}_{p h}\right)_{1}$ are the fuzzy temperatures of phonons in the first and second node, $T_{0}^{e}$ is the electron's initial temperature and $T_{0}^{p h}$ is the phonon's initial temperature.

Taking into account equations (30) and (31), the set of four fuzzy partial differential equations is obtained

$$
\left\{\begin{array}{l}
\frac{\partial \tilde{e}_{e 1}}{\partial t}+v_{e} \frac{\partial \tilde{e}_{e 1}}{\partial x}=-\frac{\tilde{e}_{e 1}-\tilde{e}_{e 1}^{0}}{\tilde{\tau}_{e}}+\tilde{Q}_{e} \\
\frac{\partial \tilde{e}_{e 2}}{\partial t}-v_{e} \frac{\partial \tilde{e}_{e 2}}{\partial x}=-\frac{\tilde{e}_{e 2}-\tilde{e}_{e 2}^{0}}{\tilde{\tau}_{e}}+\tilde{Q}_{e} \\
\frac{\partial \tilde{e}_{p h 1}}{\partial t}+v_{p h} \frac{\partial \tilde{e}_{p h 1}}{\partial x}=-\frac{\tilde{e}_{p h 1}-\tilde{e}_{p h 1}^{0}}{\tilde{\tau}_{p h}}+\tilde{Q}_{p h} \\
\frac{\partial \tilde{e}_{p h 2}}{\partial t}-v_{p h} \frac{\partial \tilde{e}_{p h 2}}{\partial x}=-\frac{\tilde{e}_{p h 2}-\tilde{e}_{p h 2}^{0}}{\tilde{\tau}_{p h}}+\tilde{Q}_{p h}
\end{array}\right.
$$

Introducing discretizing form, time and position derivatives may be written as follows [13]: 


$$
\left\{\begin{array}{l}
\left(\tilde{e}_{e 1}\right)_{i+1}^{f+1}=\left(1-\frac{\Delta t}{\tilde{\tau}_{e}}\right)\left(\tilde{e}_{e 1}\right)_{i}^{f}+\frac{\Delta t}{\tilde{\tau}_{e}}\left(\tilde{e}_{e 1}^{0}\right)_{i}^{f}+\Delta t \tilde{Q}_{e} \\
\left(\tilde{e}_{e 2}\right)_{i-1}^{f+1}=\left(1-\frac{\Delta t}{\tilde{\tau}_{e}}\right)\left(\tilde{e}_{e 2}\right)_{i}^{f}+\frac{\Delta t}{\tilde{\tau}_{e}}\left(\tilde{e}_{e 2}^{0}\right)_{i}^{f}+\Delta t \tilde{Q}_{e} \\
\left(\tilde{e}_{p h 1}\right)_{i+1}^{f+1}=\left(1-\frac{\Delta t}{\tilde{\tau}_{p h}}\right)\left(\tilde{e}_{p h 1}\right)_{i}^{f}+\frac{\Delta t}{\tilde{\tau}_{p h}}\left(\tilde{e}_{p h 1}^{0}\right)_{i}^{f}+\Delta t \tilde{Q}_{p h} \\
\left(\tilde{e}_{p h 2}\right)_{i-1}^{f+1}=\left(1-\frac{\Delta t}{\tilde{\tau}_{p h}}\right)\left(\tilde{e}_{p h 2}\right)_{i}^{f}+\frac{\Delta t}{\tilde{\tau}_{p h}}\left(\tilde{e}_{p h 2}^{0}\right)_{i}^{f}+\Delta t \tilde{Q}_{p h}
\end{array}\right.
$$

The total energy density for electrons and phonons is defined as the sum of discrete energy densities in all the lattice directions and takes the form

$$
\begin{aligned}
& \left(\tilde{e}_{e}\right)_{i}^{f+1}=\left(\tilde{e}_{e 1}\right)_{i}^{f+1}+\left(\tilde{e}_{e 2}\right)_{i}^{f+1} \\
& \left(\tilde{e}_{p h}\right)_{i}^{f+1}=\left(\tilde{e}_{p h 1}\right)_{i}^{f+1}+\left(\tilde{e}_{p h 2}\right)_{i}^{f+1}
\end{aligned}
$$

The equilibrium electron energy density and phonon energy density is the same in all lattice directions and can be calculated using the formula [26]

$$
\begin{gathered}
\left(\tilde{e}_{e}^{0}\right)_{i}^{f+1}=\frac{\left(\tilde{e}_{e}\right)_{i}^{f+1}}{2} \\
\left(\tilde{e}_{p h}^{0}\right)_{i}^{f+1}=\frac{\left(\tilde{e}_{p h}\right)_{i}^{f+1}}{2}
\end{gathered}
$$

After subsequent computations the fuzzy lattice temperature is determined using the formula

$$
\begin{aligned}
& \left(\tilde{T}_{e}\right)_{i}^{f}=\sqrt{\tilde{e}_{e}\left[\left(\tilde{T}_{e}\right)_{i}^{f}\right] /\left(n_{e} \frac{\pi^{2}}{2} \frac{k_{b}^{2}}{\varepsilon_{F}}\right)} \\
& \left(\tilde{T}_{p h}\right)_{i}^{f}=\sqrt[4]{\tilde{e}_{p h}\left[\left(\tilde{T}_{p h}\right)_{i}^{f}\right] \Theta_{D}^{3} / 9 \eta k_{b} \int_{0}^{\Theta_{D} /\left(\tilde{T}_{p h}\right)_{i}^{f-1}} \frac{z^{3}}{\exp (z)-1} \mathrm{~d} z}
\end{aligned}
$$




\section{Numerical examples}

As a numerical example the heat transport in a gold thin film of the dimensions $200 \mathrm{~nm}$ has been analyzed. The following input data have been introduced: the relaxation time $\tilde{\tau}_{r e}=(0.038,0.04,0.042) \mathrm{ps}, \tilde{\tau}_{r p h}=(0.76,0.8,0.84) \mathrm{ps}$, the Debye temperature $\Theta_{D}=170 \mathrm{~K}$, the coupling factor $\mathrm{G}=2.3 \cdot 10^{16} \mathrm{~W} /\left(\mathrm{m}^{3} \mathrm{~K}\right)$, the external heat source $Q^{\prime}=10^{20} \mathrm{~W} / \mathrm{m}^{3}$, the fuzzy boundary conditions of the $1^{\text {st }}$ type on the left boundary $\tilde{T}_{b 1}^{e}=\tilde{T}_{b 1}^{p h}=(285,300,315) \mathrm{K}$ and the $2^{\text {nd }}$ type on the right boundary $\tilde{q}_{b 2}^{e}=\tilde{q}_{b 2}^{p h}=\tilde{0}$, the initial temperature $T_{0}=300 \mathrm{~K}$. The lattice step $\Delta x=20 \mathrm{~nm}$ and the time step $\Delta t=0.01 \mathrm{ps}$ have been assumed.

Figures 2 and 3 illustrate the fuzzy temperature distribution of electrons in the thin gold film for the chosen times and chosen values of parameter $\alpha$. Figure 4 presents the interval values of temperatures for the chosen parameter $\alpha$ at the node corresponding to the depth of $60 \mathrm{~nm}$ after $0.2 \mathrm{ps}$.

In the Figures 5 and 6 the heating curves at the internal nodes $1(40 \mathrm{~nm})$, $2(60 \mathrm{~nm})$ and $3(80 \mathrm{~nm})$ for chosen values of the parameter $\alpha$ are shown.

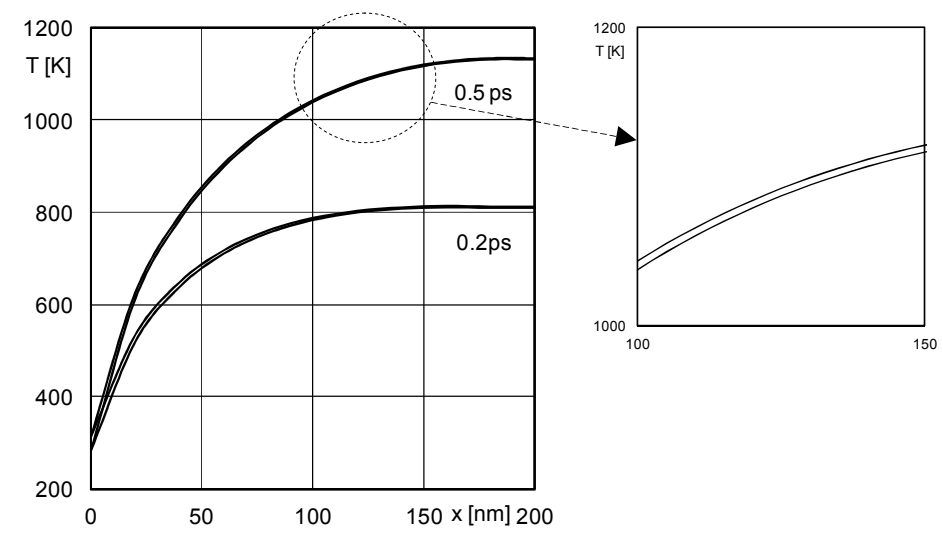

Fig. 2. The fuzzy temperature distribution for $\alpha=0$

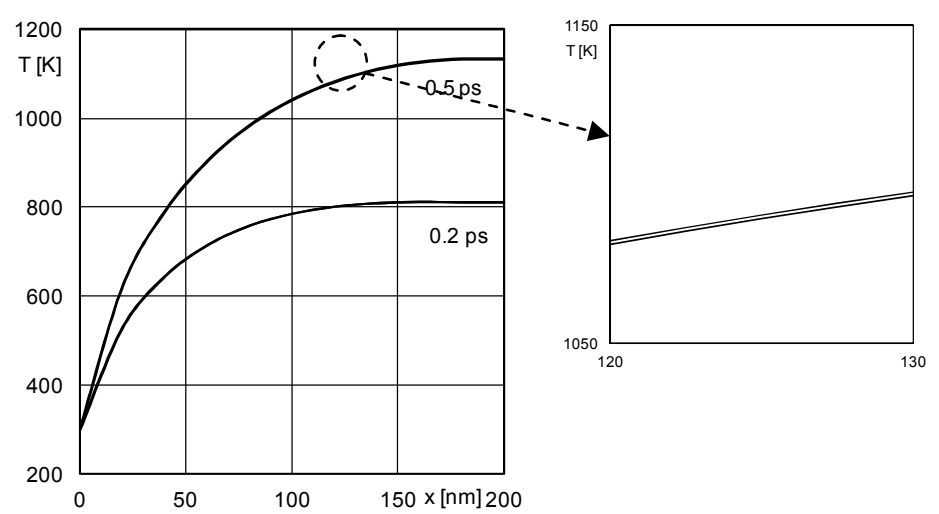

Fig. 3. The fuzzy temperature distribution for $\alpha=0.75$ 


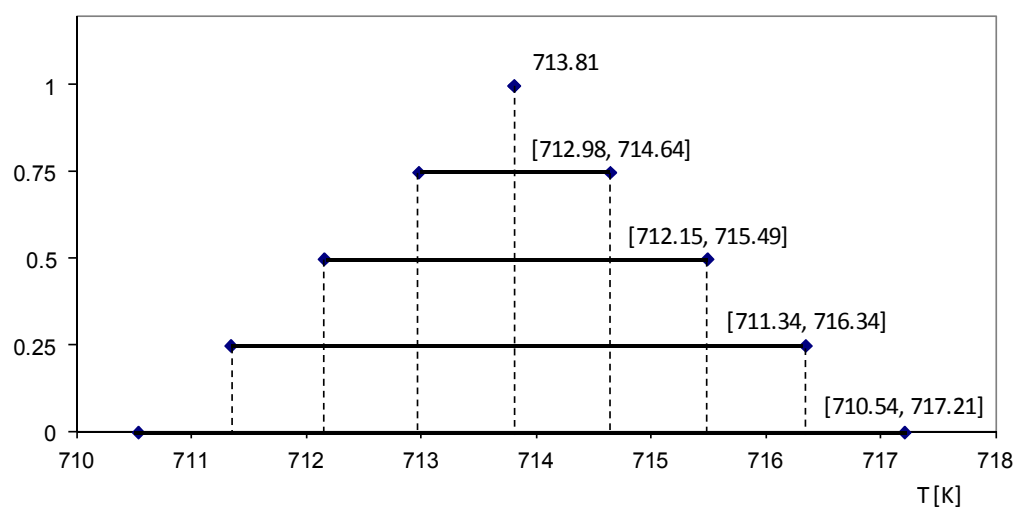

Fig. 4. The interval values of temperatures for the chosen parameter $\alpha$ at the node $60 \mathrm{~nm}$ after $0.2 \mathrm{ps}$

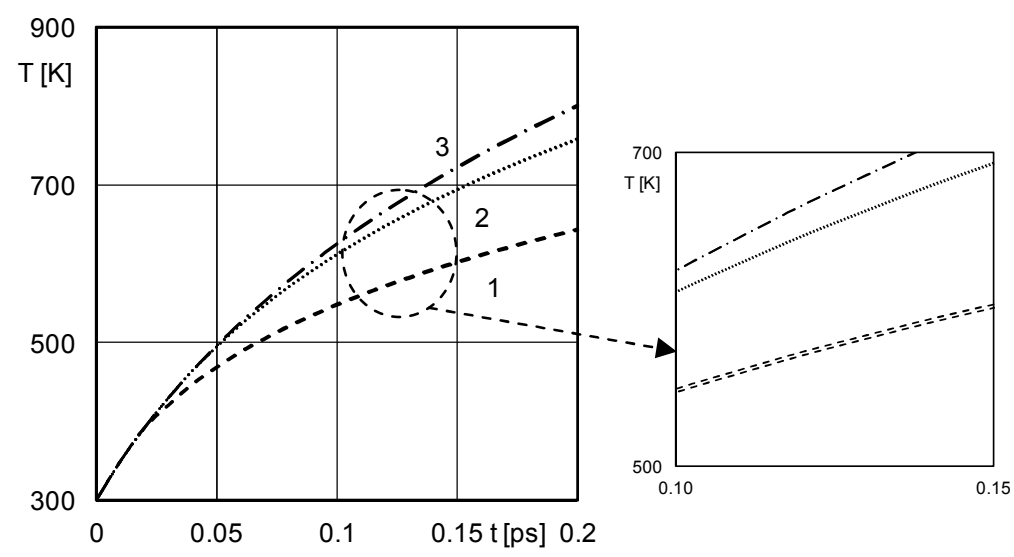

Fig. 5. Heating curves for $\alpha=0.75$ at the chosen nodes

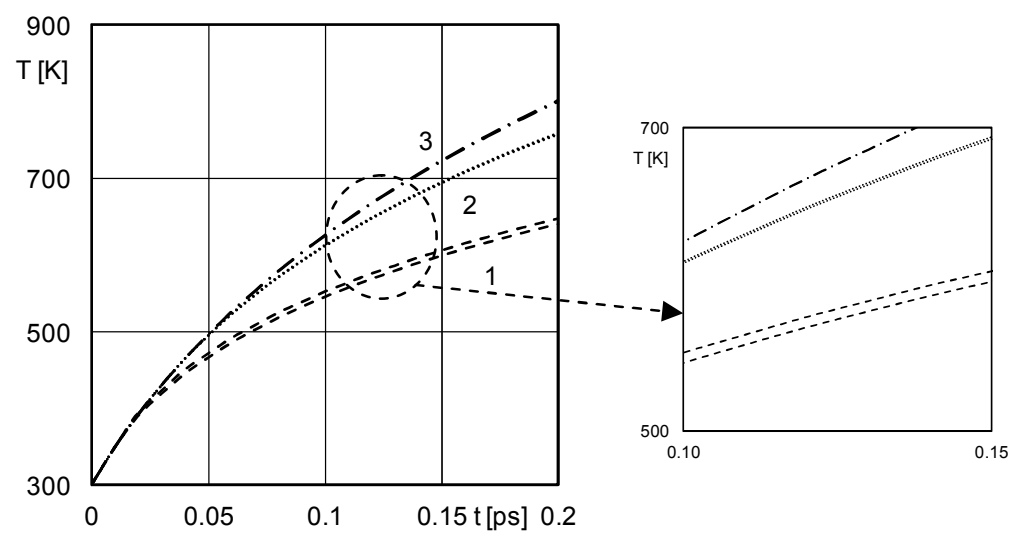

Fig. 6. Heating curves for $\alpha=0.25$ at the chosen nodes 


\section{Conclusions}

In the paper the Boltzmann transport equation with the fuzzy values of the relaxation times and the boundary conditions has been considered. The fuzzy version of the lattice Boltzmann method with $\alpha$-cuts for solving 1D problems has been presented.

Such an approach allows one to avoid complicated fuzzy arithmetic and treat the considered fuzzy numbers as interval numbers. For bigger values of $\alpha$, the temperature interval is narrower. For $\alpha=1$, the wideness of the temperature interval is equal to 0 .

The generalization of LBM allows one to find the numerical solution in the fuzzy form and such information may be important especially for the parameters which are estimated experimentally, for example the relaxation time.

\section{Acknowledgement}

This work is supported by the project BK-255/RMT4/2016.

\section{References}

[1] Zhang Z.M., Nano/Microscale Heat Transfer, McGraw-Hill, New York 2007.

[2] Chen G., Borca-Tasciuc D., Yang R.G., Nanoscale heat transfer, [in:] Encycl. of Nanoscience and Nanotechnology, CA: American Scientific Publishers, Valencia, 7, 2004, 429-359.

[3] Smith A.N., Norris P.M., Microscale heat transfer, [in:] Heat Transfer Handbook, eds. A. Bejan, D. Kraus, John Wiley \& Sons, 2003, Ch.18, 1309-1358.

[4] Cattaneo C., A form of heat conduction equation which eliminates the paradox of instantaneous propagation, Comp. Rend. 1958, 247, 431-433.

[5] Majchrzak E., Mochnacki B., Greer A.L., Suchy J.S., Numerical modeling of short pulse laser interactions with multi-layered thin metal films, CMES: Computer Modeling in Engineering and Sciences 2009, 41, 2, 131-146.

[6] Majchrzak E., Kałuża G., Heat flux formulation for 1D dual-phase lag equation, Journal of Applied Mathematics and Computational Mechanics 2015, 14(1), 71-78, DOI: 10.17512/ jamcm.2015.1.07.

[7] Majchrzak E., Mochnacki B., Sensitivity analysis of transient temperature field in microdomains with respect to the dual phase lag model parameters, International Journal for Multiscale Computational Engineering 2014, 12(1), 65-77, DOI: 10.1615/IntJMultCompEng. 2014007815.

[8] Mochnacki B., Paruch M., Estimation of relaxation and thermalization times in microscale heat transfer model, Journal of Theoretical and Applied Mechanics 2013, 51, 4, 837-845.

[9] Majchrzak E., Dziatkiewicz J., Analysis of ultashort laser pulse interactions with metal films using a two-temperature model, Journal of Applied Mathematics and Computational Mechanics 2015, 14(2), 31-39. DOI: 10.17512/jamcm.2015.2.04.

[10] Dziatkiewicz J., Kuś W., Majchrzak E., Burczyński T., Turchan Ł., Bioinspired identification of parameters in microscale heat transfer, International Journal for Multiscale Computational Engineering 2014, 12(1), 79-89.

[11] Tzou D.Y., Macro- to Microscale Heat Transfer: The Lagging Behavior, Taylor and Francis, 1997. 
[12] Piasecka-Belkhayat A., Korczak A., Application of the interval lattice Boltzmann method for a numerical modeling of thin metal films irradiation by ultra short laser pulses, IAPGOŚ 2014, 4, 85-88.

[13] Piasecka Belkhayat A., Korczak A., Modelling of transient heat transport in one-dimensional crystalline solids using the interval lattice Boltzmann method, [in:] Recent Advances in Computational Mechanics, eds. T. Łodygowski, J. Rakowski, P. Litewka, Taylor \& Francis Group, A Balkema Book, London 2014, 363-368.

[14] Mochnacki B., Piasecka Belkhayat A., Numerical modeling of skin tissue heating using the interval finite difference method, MCB: Molecular \& Cellular Biomechanics 2013, 10, 3, 233-244.

[15] Escobar R.A., Ghai S.S., Jhon M.S., Amon C.H., Multi-length and time scale thermal transport using the lattice Boltzmann method with application to electronics cooling, Journal of Heat and Mass Transfer 2006, 49, 97-107.

[16] Pisipati S., Geer J., Sammakia B., Murray B.T., A novel alternate approach for multiscale thermal transport using diffusion in the Boltzmann Transport Equation, International Journal of Heat and Mass Transfer 2011, 54, 3406-3419.

[17] Ashcroft N.W., Mermin N.D., Solid State Physics, Harcourt College Publishers, New York 1976.

[18] Venkatakrishnan K., Tan B., Ngoi B.K.A., Femtosecond pulsed laser ablation of thin gold film, Optics \& Laser Technology 2002, 34, 199-202.

[19] Hanss M., Applied Fuzzy Arithmetic, Springer-Verlag, Berlin-Heidelberg 2005.

[20] Otto K., Lewis A.D., Antonsson E., Approximation alpha-cuts with the vertex method, Fuzzy Sets and Systems 1993, 55, 43-50.

[21] Giachetti R.E., Young R.E., A parametric representation of fuzzy numbers and their arithmetic operators, Fuzzy Sets and Systems 1997, 91, 185-202.

[22] Guerra M.L., Stefanini L., Approximate fuzzy arithmetic operations using monotonic interpolations, Fuzzy Sets and Systems 2005, 150, 5-33.

[23] Piasecka Belkhayat A., Interval boundary element method for imprecisely defined unsteady heat transfer problems, D.Sc. Dissertation, Gliwice 2011.

[24] Ghai S.S., Kim W.T., Escobar R.A., et al., A novel heat transfer model and its application to information storage systems, Journal of Applied Physics 2005, 97, 10 P703.

[25] Lee J.B., Kang K., Lee S.H., Comparison of theoretical models of electron-phonon coupling in thin gold films irradiated by femtosecond pulse lasers, Materials Transactions 2011, 52, 3, 547-553.

[26] Escobar R., Smith B., Amon C., Lattice Boltzmann modeling of subcontinuum energy transport in crystalline and amorphous microelectronic devices, Journal of Electronic Packaging 2006, $128(2), 115-124$. 